\title{
Study on Storage life predicting Method of Missile equipment
}

\author{
LI Ke, SHI Lian-yan, HE Xin, JIANG Huixia
}

4th Department, Shijiazhuang Mechanical engineering college, Shijiazhuang, HEBEI, CHINA, 050003

Ken_like@126.com

Keywords: storage reliability; storage life predicting method; missile equipment; data "mutation"

\begin{abstract}
In the paper, the mathematical statistical method is adopted to build a storage life prediction model of missile equipment. Data "mutation" and confidence area appeared in the process of the model parameters determining and life predicting are discussed, and several improved decoding algorithms for inefficient distribution parameters are analyzed by simulation.

The results show that the $\chi^{2}$ optimizing method is a better fitting algorithm for storage life prediction of missile equipment.
\end{abstract}

\section{Introduction}

Missile equipment is a sort of weapon, which is stored for a long time and one-use; it always is in the stored state during most of the time from its production to usage. Storage life of the missile refers to the promised time span during which a missile could be stored under certain environmental conditions, the performance and reliability of the missile in this period could still meet the requirements of regulations. Accurate prediction of the safe storage life of a missile could effectively keep harmony among repository quantity, repository time and maintenance, and then could predict the regular maintenance time of a missile; so accurate prediction of the safe storage life of a missile has important significance for the repairing, maintenance and safe use of weapon equipment. In the paper, the relevant data of a missile is analyzed by using the mathematical statistical method, and the storage life prediction is carried out based on the reliability model.

\section{Determination of inefficient distribution}

\subsection{Distribution types}

Classic types of inefficient distribution of storage life of a missile mainly have three [1]:

(1) Weibull distribution

$F_{(t)}=1-\exp \left(a t^{b}\right)$

Inverse weibull distribution

$F_{(t)}=\exp \left(a t^{b}\right)$

(3) Minimal value distribution

$F_{(t)}=1-\exp [-\exp (a t-b)]$

\subsection{Parameters estimation}

It is a very complicated and important job to estimate the parameters of inefficient distribution by using sample values in the study of storage reliability of a missile. The methods commonly used to estimate the parameters include the probability map method, regression analysis method, moment estimation method, least squares method, maximum likelihood method, genetic algorithm, etc. A lot of reliability engineering practices show that the maximum likelihood estimation method has higher precision $[2,3]$ 。

For the above three kinds of inefficient distribution, maximum likelihood function is:

$$
L=C \prod_{i=1}^{m} F\left(t_{i}\right)^{Y_{i}}\left[1-F\left(t_{i}\right)\right]^{n_{i}}
$$

In the formula, ri is represented for the reliability of the ith sample, ni is represented for the size 
of the ith sample, the symbols in the follow formulas have the same meaning.

(1) For weibull distribution

$$
\ln L=\sum_{i=1}^{m} r_{i} \ln \left(1-e^{a t_{i}^{b}}\right)+\sum_{i=1}^{m} a t_{i}^{b} n_{i}
$$

Assumed: $\frac{\partial \ln L}{\partial a}=0, \frac{\partial \ln ^{i=1} L}{\partial b}=0$, then a simultaneous likelihood equations about a and b could be got:

$$
\left\{\begin{array}{l}
\sum_{i=1}^{m} r_{i} \frac{-t_{i}^{b} e^{a t_{t}^{b}}}{1-e^{a t_{i}^{b}}}+\sum_{i=1}^{m} n_{i} t_{i}^{b}=0 \\
\sum_{i=1}^{m} r_{i} \frac{-a t_{i}^{b}\left(\ln t_{i}\right) e^{a t_{i}^{b}}}{1-e^{a t_{i}^{b}}}+\sum_{i=1}^{m} a n_{i} t_{i}^{b} \ln t_{i}^{b}=0
\end{array}\right.
$$

(2) For inverse weibull distribution

$$
\ln L=\sum_{i=1}^{m} a t_{i}^{b} r_{i}-\sum_{i=1}^{m} n_{i} \ln \left(1-e^{a t_{i}^{b}}\right)
$$

The simultaneous likelihood equations are as follows:

$$
\left\{\begin{array}{l}
\sum_{i=1}^{m} n_{i} \frac{-t_{i}^{b} e^{a t_{i}^{b}}}{1-e^{a t_{i}^{b}}}+\sum_{i=1}^{m} r_{i} t_{i}^{b}=0 \\
\sum_{i=1}^{m} n_{i} \frac{-a t_{i}^{b}\left(\ln t_{i}\right) e^{a t_{i}^{b}}}{1-e^{a t_{i}^{b}}}+\sum_{i=1}^{m} a r_{i} t_{i}^{b} \ln t_{i}^{b}=0
\end{array}\right.
$$

(3)For minimal value distribution

$$
\ln L=\sum_{i=1}^{m} r_{i} \ln [1-\exp (-\exp (a t+b))]-\sum_{i=1}^{m} e^{a t_{i}+b} n_{i}
$$

The simultaneous likelihood equations are as follows:

$$
\left\{\begin{array}{l}
\sum_{i=1}^{m} r_{i} \frac{-t_{i} e^{a t_{i}+b} e^{-e^{a_{i}+b}+b}}{1-e^{-a t_{i}^{b}+b}}-\sum_{i=1}^{m} n_{i} t_{i} e^{a_{i}+b}=0 \\
\sum_{i=1}^{m} r_{i} \frac{-e^{a t_{i}+b} e^{-e^{a_{i}}+b}}{1-e^{-a t_{i}^{b}+b}}+\sum_{i=1}^{m} n_{i} e^{a t_{i}+b}=0
\end{array}\right.
$$

The parameters ri and ni in above equations are known values, the parameters a and $b$ are parameters to be calculated. The traditional method to calculate the parameters $a$ and $b$ is the iterative method.

\section{Improved algorithm of parameter estimation}

The maximum likelihood estimating method is used to calculate the simultaneous transcendental equations, and the procedure of the method is complex and the calculation is large. In the paper, two improved methods are applied to make the parameter calculation simple and accurate.

\subsection{Optimizing method of the likelihood function}

According to the formula (6), the likelihood function obtains the maximum value at the parameter estimating point, so there is:

$$
\min F(\theta)=-\ln L(\theta)=-\sum_{i=1}^{n} \ln f\left(x_{i} ; \theta\right)
$$

That is to say, the minus likelihood function has a minimum. In term of the idea of optimization, when the function gets a minimal value, it becomes very simple to calculate unknown parameters.

There are many optimizing methods, the fminsearch function is selected to solve the problem, which has a fast convergent rate, high accuracy and stability of result; it could simultaneously calculate the values of unknown variables of the objective function.

The objective function has only two unknown variables a and b, and they are easy to be calculated with one optimizing method. The parameter values are not only calculated, but also the value of the likelihood function is calculated by optimizing method of the likelihood function, and it is identified as inefficient distribution while the likelihood function gets the minimal value. 


\section{$3.2 \chi^{2}$ optimizing method}

The oldest method for fitting checkout of fine degrees is the minimal $\chi^{2}$ checkout. If only a distribution makes the $\chi^{2}$ value reach a minimum, it is considered as the best distribution. Set up a statistical variable:

$$
\chi^{2}=-\sum_{i=1}^{n} \frac{n_{i}\left(F(t)-p_{i}\right)^{2}}{F(t)\left(1-F\left(t_{i}\right)\right)}
$$

In the equation, $\hat{p}_{i}=r_{i} / n_{i}$ and it can be determined by the known values.

In fact the idea of $\chi^{2}$ optimizing method is very simple, it just inverses calculating process of iteration method. Iteration method firstly assumes a distribution types, and then solves a simultaneous transcendental equations to calculate parameters, finally substitute it into formula (11) to test, one distribution is identified as inefficient distribution as the $\chi^{2}$ gets a minimal value. But $\chi^{2}$ optimizing method firstly assumes that formula (11) had a minimum, meanwhile an optimizing calculation is performing to determine parameter values, and get $\chi^{2}$ value at the same time. It is when the $\chi^{2}$ gets a minimum value that one distribution will be considered as inefficient distribution. The $\chi^{2}$ optimizing method solves parameters with fminsearch function to calculate. The biggest advantage of $\chi^{2}$ optimizing method is that it has little intermediate variables, requirement for the initial value is not high, for example taking $[0,0]$ can get satisfactory results, stability of the results is robust, and accuracy is very high.

\section{Prediction of storage life}

\subsection{Evaluation of storage reliability}

(l) For weibull distribution

$$
R_{(t)}=\exp \left(a t^{b}\right)
$$

The storage reliability of the $\mathrm{t}$ moment is as follow:

$$
R_{S(t)}=R_{(t)} R_{0}=R_{0} \exp \left(a t^{b}\right)
$$

$R_{0}$ is the inherent reliability, which could be determined by data of products when they leave the factory.

(2) For inverse weibull distribution

$$
R_{(t)}=1-\exp \left(a t^{b}\right)
$$

The storage reliability of the t moment is as follow:

$$
R_{s(t)}=R_{(t)} R_{0}=R_{0}\left[1-\exp \left(a t^{b}\right)\right]
$$

(3) For minimal value distribution

The storage reliability of the $\mathrm{t}$ moment is as follow:

$$
R_{S(t)}=R_{(t)} R_{0}=R_{0} \exp [-\exp (a t+b)]
$$

\subsection{Prediction of storage life}

When a reliable level RL is set, the relevant reliable storage life denoted as $t_{R_{L}}$ could be calculated through function $\mathrm{R}(\mathrm{t})$, which is called a reliable point estimation of storage life. Obviously, reliable storage life is associated with a presumed value RL, the smaller a RL, the longer the reliable storage life, the greater a RL, the shorter the reliable storage life.

(l) For weibull distribution, the point estimation of the reliable storage life could be established through by the equation (14):

$$
t_{R_{L}}=\left(\frac{\ln R_{L}}{a}\right)^{\frac{1}{b}}
$$

(2) For the inverse Weibull distribution, likewise by equation (16):

$$
t_{R_{L}}=\left(\frac{\ln \left(1-R_{L}\right)}{a}\right)^{\frac{1}{b}}
$$

(3) For minimal value distribution, likewise by equation (17): 


$$
t_{R_{L}}=\frac{1}{a}\left(\ln \ln \frac{1}{R_{L}}-b\right)
$$

\section{Simulating results}

\subsection{Procession on data "mutation"}

The overall unreliability of the $t_{i}$ moment is $\mathrm{pi}$, and in terms of characteristics of storage reliability of the missile, there is an inequality as follow:

$$
p_{i}<p_{1}<\cdots p_{m}
$$

But due to randomness of samples, especially when the size of samples is small, formula (21) may not set up. The phenomenon pi-1>pi is named as "reverse sequence" or "data mutation". The "data mutation" is abnormal from the objective laws that the unreliability of missile should be increased with the extension of storage time, so "data mutation" should be adjusted to make samples into a non-decreasing sequence [4]。

Bayes estimating method is applied, and the idea is as: at moment $t_{i}$, unreliability of samples denoted as $p_{i}=\left(\frac{f_{i}+1}{c-1}\right) \ln \left(\frac{n+c+1}{n+2}\right)$ ( $\mathrm{C}$ is assumed as 4), a "data mutation" is discovered and assumed that it belong to a time interval [tj, ti], the more reliable end of the two ends of the interval is selected as the referenced point. If ti is more reliable than $t j$, it is taken as the referenced point, and then revised forward step by step based on pi to obtain the Bayes estimation of pi-1:

$$
\hat{p}_{i-1}=\frac{\int_{p_{-1}}^{p_{i}} p^{f_{t-1}+0.5}(1-p)^{n_{t-1}-f_{t-1}} d p}{\int_{p_{p_{-1}}}^{p_{i}} p^{f_{t_{1-1}}-0.5}(1-p)^{n_{1-1}-f_{t-1}} d p}
$$

On the contrary, If $\mathrm{t} j$ is more reliable than ti, it should be taken as the referenced point, and then revised backward step by step to obtain the Bayes estimation of $\mathrm{pj}+1$ :

$$
\hat{p}_{j+1}=\frac{\int_{p_{j}}^{p_{j+1}} p^{f_{j+1}+0.5}(1-p)^{n_{j+1}-f_{j+1}} d p}{\int_{p_{j}}^{p_{t+1}} p^{f_{j+1}-0.5}(1-p)^{n_{j+1}-f_{j+1}} d p}
$$

\subsection{Numerical simulation}

\subsubsection{Data preparation}

Table 1 is data of one missile storage reliability [5]。

Table 1: A certain type of missile reliability data

\begin{tabular}{c|c|c|c|c|c|c|c|c|c}
\hline Sample number i & 1 & 2 & 3 & 4 & 5 & 6 & 7 & 8 & 9 \\
\hline $\begin{array}{c}\text { storage time } \\
\text { (month) }\end{array}$ & 0 & 28 & 40 & 50 & 60 & 70 & 73 & 82 & 87 \\
\hline $\begin{array}{c}\text { sample size } \\
\text { (shot) }\end{array}$ & 300 & 100 & 150 & 120 & 150 & 200 & 180 & 200 & 200 \\
\hline inefficient number (shot) & 3 & 1 & 3 & 4 & 8 & 10 & 8 & 16 & 18 \\
\hline Unreliability $\left(\mathrm{p}_{\mathrm{i}}\right)$ & 0.034983 & 0.004951 & 0.039934 & 0.042397 & 0.053178 & 0.077363 & 0.069337 & 0.082289 & 0.097264 \\
\hline
\end{tabular}

In table 1, p2, p7 produced "upside down", and the data is corrected using the foregoing method.

\subsubsection{Parameter estimation}

The results of the parameter estimation of iteration method, the likelihood optimizing method and $\chi^{2}$ optimizing method is shown as table 2, when the minimal distribution function is adopted different initial conditions. 
Table 2. calculate results omparison of three parameters estimation method

\begin{tabular}{|c|c|c|c|c|}
\hline initial & value & Iterative method & $\begin{array}{c}\text { Likelihood } \\
\text { optimizing method }\end{array}$ & $\chi^{2}$ optimizing method \\
\hline$[0.1$ & $-5.0]$ & $\begin{array}{c}a=0.668937 \\
b=-4.275405\end{array}$ & $\begin{array}{l}a=0.609389 \\
b=-6.35934\end{array}$ & $\begin{array}{c}a=0.597831 \\
b=-5.354262\end{array}$ \\
\hline$[0.09$ & $-4.2]$ & $\begin{array}{l}a=0.633300 \\
b=-5.51878\end{array}$ & $\begin{array}{c}a=0.598451 \\
b=-5.341850\end{array}$ & $\begin{array}{c}a=0.597831 \\
b=-5.354262\end{array}$ \\
\hline$[0.09$ & $-4.86]$ & $\begin{array}{c}a=0.497704 \\
b=-5.833990\end{array}$ & $\begin{array}{l}a=0.509389 \\
b=-6.181283\end{array}$ & $\begin{array}{l}a=0.629912 \\
b=-6.778443\end{array}$ \\
\hline \multicolumn{2}{|c|}{ Very poor } & $\begin{array}{l}a=0.071233 \\
b=1.017495\end{array}$ & $\begin{array}{l}a=0.109839 \\
b=1.017459\end{array}$ & $\begin{array}{l}a=0.000675 \\
b=0.004184\end{array}$ \\
\hline
\end{tabular}

Due to different degree of dependence on initial values accuracy, likelihood optimization may get local optimal solution, also get the global optimal solution, but $\chi^{2}$ optimization method gets the global optimal solution. The results of fitting checkout test also show that the minimal value distribution is better than the weibull distribution and inverse weibull distribution.

\subsubsection{Calculation of storage life}

The reliability function of the minimal value distribution is $R_{(t)}=\exp [-\exp (a t+b)]$, the results(a $=$ $0.629912, \mathrm{~b}=6.778443)$ of the $\chi^{2}$ optimizing method are used as parameter values of $\mathrm{R}(\mathrm{t})$, so the reliability function is:

$$
R_{(t)}=\exp \left[-\exp \left(\frac{t-10.760936}{1.587523}\right)\right]
$$

The inefficient distribution function is as follow:

$$
F_{(t)}=1-\exp \left[-\exp \left(\frac{t-10.760936}{1.587523}\right)\right]
$$

When a confidence level $\mathrm{r}$ is set, the storage reliability $\mathrm{Rs}(\mathrm{t})$ of missiles could be calculated during a given storage time, and the storage life of lower limit $t_{R_{\iota}}$ with one reliability level is calculated too. For example table 3.

Table 3. Storage reliability point estimation and confidence limit if confidence level $r$ is 0.90

\begin{tabular}{|c|c|c|c|c|}
\hline Reliable level & 0.8 & 0.85 & 0.9 & 0.95 \\
\hline Reliable storage life point estimation (years) & $>9$ & 8.4 & 7.9 & 6.4 \\
\hline
\end{tabular}

According to table 3, even confidence level $\mathrm{r}$ is 0.90 , missiles are stored after eight years and five months; the lower limit value of reliability is not less than 0.85 . So it could be predicted that the reliable storage life of the missile equipment is more than 8 years and 5 months.

\section{Conclusions}

The paper adopts the method of mathematical statistics, and applies the Bayes estimating method to solve the problem of "data mutation" based on the contrastive analysis of several typical distribution functions. The Minimal distribution function is selected as the inefficient function of missile equipment storage. The $\chi^{2}$ optimizing method is applied to implement parameter estimation, and storage life of one missile equipment is predicted based on the inefficient distribution.

\section{References}

[1] PAN Wen-geng. The Failure Mechainism Analysis of Long-stored Amminition [J], Journal of Projectiles,Rockets,Missiles and Guidance, 2007, 27(l):122 125.

[2] ZHOU Guoli. PROBABILITY AND STATISTICS, CHONGQING: CHONGQING UNIVERSITY PRESS [M], 2004, 3. 
[3] HE Shuyuan. PROBABILITY, BEIJING: BEIJING UNIVERSITY PRESS [M]， 2006,1.

[4] LI Changfu, XIA Jianzhong. Study on Ammunition Storage Reliability and Evaluating Method with Deleting Data [J], ACTA ARMAMENTRII, 1996, 17(4) : 303 307.

[5] ZHANG Zhihui. Study on Plateau Climate of Ammunition Storage Reliability and Evaluating Method [D], NANJING UNIVERSITY OF SCIENCE AND TECHNOLOGY, 2008.6. 